\title{
Validation of the French version of the Functional, Communicative and Critical Health Literacy scale (FCCHL)
}

Youssoufa M. Ousseine ${ }^{1}$, Alexandra Rouquette ${ }^{2,3}$, Anne-Déborah Bouhnik ${ }^{1}$, Laurent Rigal ${ }^{3}$, Virginie Ringa ${ }^{2}$, Allan 'Ben' Smith' ${ }^{4,5}$ and Julien Mancini' ${ }^{1,6^{*}}$

\begin{abstract}
Background: Health literacy is a key asset, defined as the capacity to acquire, understand and use information in ways which promote and maintain good health.

Objectives: To assess the reliability and validity of the French translation of the Functional, Communicative and Critical Health Literacy (FCCHL) scale.

Methods/participants: A cross-sectional survey using an online questionnaire was proposed to all members of Seintinelles association. Exploratory and confirmatory factorial analyses were conducted.

Results: Data from 2342 respondents (45.8\% had cancer history) were analysed. The FCCHL scale was well-accepted (missing value by item $\leq 0.7 \%$ ). Factor analysis revealed an acceptable fit of three-factor model (comparative fit index $=0$. 922, root mean square error of approximation $=0.065$ and standardized root mean square residual $=0.052$ ). The FCCHL showed satisfactory reliability $(\alpha=0.77)$ and scalar invariance was reached for education and deprivation, but not for age. Known group validity was verified as mean scale scores differed according to education, deprivation and age, as expected. Conclusion: The French version of the FCCHL provides a brief reliable and valid measure to explore the dimensions of health literacy. It could be used by health professionals to screen for health literacy level in order to develop this skill and to tailor health communication.
\end{abstract}

Keywords: Health literacy, Measurement, Validation studies, Psychometrics, Cancer, France

\section{Introduction}

Health literacy (HL) is a key asset, defined as "the individuals' capacity to obtain, process and understand basic health information and services needed to make appropriate health decisions" [1, 2]. Consistently, authors like Nutbeam [3] distinguish three skills: functional literacy, which includes basic skills in reading and writing necessary to understand health information; communicative literacy, which corresponds to the necessary advanced skills to communicate or interact with the healthcare system; and critical literacy to analyse the information

\footnotetext{
* Correspondence: julien.mancini@univ-amu.fr

'Aix-Marseille Univ, INSERM, IRD, UMR912, SESSTIM, Institut Paoli-Calmettes,

"Cancers, Biomedicine \& Society" group, 232, Bd Ste Marguerite, BP 156,

13273 Marseille Cedex 9, France

${ }^{6}$ APHM, Timone Hospital, Public Health Department (BIOSTIC), Marseille,

France

Full list of author information is available at the end of the article
}

obtained to act at best. Accordingly, the Functional, Communicative and Critical Health Literacy scale (FCCHL) measures all three distinguished dimensions of HL [4]. A generic tool [5] was adapted from the first version developed to specifically evaluate $\mathrm{HL}$ in diabetic patients [4] and has been validated in several populations including Dutch/German citizens [6-8], and Australian Adolescents and Young Adults with cancer [9]. This brief instrument (14-items) has demonstrated relevance to patients and ease of administration [9]. However, no validation of FCCHL exists in French.

Validated translations of HL measures are needed, as a growing literature has shown the importance of evaluating HL in both patients and general population. Indeed, limited HL predicts poorer health and has demonstrated associations with several patient-reported outcomes, 
such as poorer health-related quality of life and more mental distress among cancer patients [10].

Our aim was the psychometric evaluation of the French translation of the FCCHL scale.

\section{Methods}

The FCCHL was translated from English to French by three independent researchers. The final version of each item was then chosen with the help of a bilingual psychologist. Individual cognitive interviews were then conducted with six cancer patients to evaluate the wording and understanding of the translated items. Minor refinements were then made comparing our translation with the translation from the Japanese to French made by other French researchers.

Data were collected using a self-administered online questionnaire proposed to all adult members of Seintinelles (www.seintinelles.com) between June 16th and 30th, 2016. Seintinelles is a French national association including 12,747 members (participant numbers as declared on January 15th 2016) who are cancer patients, cancer survivors and/or other people (e.g. caregivers) "wishing to help cancer research" [11]. They are mostly breast cancer patients because it was initially created based on the model of Army of Women ${ }^{\circ}$. Use of Seintinelles enabled the rapid recruitment of a large sample for psychometric validation of the French translation of the FCCHL scale. The final sample size was larger than the minimum of 500 to 800 respondents needed to perform exploratory factorial analysis for a three-factor scale with 10 to 15 items [12].

Floor or ceiling effects at the scale level were considered to be present if more than $15 \%$ of respondents achieved the lowest or highest possible score, respectively [13]. At the item level, these effects were considered to be present if more than 95\% answered the lowest or highest response category [14]. Reliability was assessed by Cronbach's alpha $(\alpha)$ with values $\alpha \geq 0.7$ considered satisfactory [15].

An exploratory factor analysis (EFA) was performed on one third of the sample, randomly selected. To assess construct validity, confirmatory factor analysis (CFA), based on the factorial structure found using EFA and in literature, was subsequently conducted on the other two-thirds of the sample. Multiple-group CFA and nested model comparisons were used to evaluate measurement invariance across age groups, education levels and deprivation using the following sequence: configural, metric and scalar invariance. The robust weighted least squares (WLSMV) estimator was used for both EFA and CFA $[16,17]$. The root mean square error of approximation (RMSEA, good fit if $<0.06$, poor fit if $\geq 0.10$, acceptable elsewhere), the comparative fit index (CFI) and the Tucker-Lewis index (CFI and TLI, good fit if $>0.95$, poor fit if $<0.90$, acceptable elsewhere) were used to examine model fit [18]. For measurement invariance, each level of invariance was considered to be met if the fit indices difference between each level was equal or less than -0.01 for $\triangle \mathrm{CFI}$ and equal or less than 0.015 for $\triangle$ RMSEA $[19,20]$. When a level of invariance was not met, non-invariant items were identified by reviewing modification indices in order to release equality constraints concerning these items until partial invariance was met.

Our a priori hypotheses were that a higher HL would be associated with higher education, younger age, French as mother language and lack of deprivation $[4,21,22]$. Student's $\mathrm{t}$ tests and ANOVAs were used to compare mean FCCHL levels.

All analyses were two-tailed and performed using SPSS PAWS Statistics 18.0 and Mplus software version 7.4. Pvalues $<0.05$ were considered significant.

\section{Results}

In June 2016, 2444 participants were surveyed after excluding 124 participants (4.8\%) who only completed their sociodemographic characteristics. Missing values by item ranged from $0.1 \%$ (FCCHL6) to $0.7 \%$ (FCCHL3 and FCCHL5) and 2342 participants (95.8\%) answered all 14 items of the FCCHL scale. The following results were obtained from participants with complete data of FCCHL $(n=2342)$.

The mean age of those 2342 participants was 47.6 years ( $\mathrm{SD}=13.6$ ), $96.4 \%$ of participants were women, $45.8 \%$ had a history of cancer and $18.1 \%$ were deprived (Table 1 ).

The lowest total score was 27 (versus a possible 14) and 32 respondents $(1.4 \%)$ had the highest score possible (70) indicating no floor or ceiling effect at the scale level. The percentages of respondents for each of the response categories range from $1 \%$ to $72 \%$ over the 14 items, indicating no floor or ceiling effects at the item level. Globally, correlations between the different items within each dimension were $>0.4$ (ranging from 0.41 to 0.76 ) (Additional file 1: Table S1).

The EFA with promax rotation revealed three factors, explaining $55 \%$ of the variance. The loading matrix obtained is shown in Table 2. Among the four items for critical HL, three clearly loaded on the third factor but the last item (FCCHL14) loaded on the second factor (0.42).

CFA indicated reasonable fit indices for a 3-factor model (with correlation between 3-factors, Fig. 1): RMSEA $=0.087$ (90\% confidence interval 0.082-0.092), CFI $=0.946$ and $\mathrm{TLI}=0.933$. When the item FCCHL14 was modelled in the second dimension, fit indices were: RMSEA $=0.086$ (90\% confidence interval $0.081-0.091$ ) and $\mathrm{CFI}=0.947$ and $\mathrm{TLI}=0.935$. A significant decrease $(3$ and 2 factor nested model test, $p<0.001)$ in model fit was observed when communicative and critical dimensions 
Table 1 Main participants' characteristics $(n=2342)$

\begin{tabular}{|c|c|c|c|}
\hline Sociodemographic and medical history & & $\mathrm{n}$ & $\%$ \\
\hline \multicolumn{4}{|l|}{ Age } \\
\hline $18-40$ & & 777 & 33.2 \\
\hline $41-60$ & & 1111 & 47.4 \\
\hline $61-83$ & & 454 & 19.4 \\
\hline Female Gender & & 2258 & 96.4 \\
\hline French maternal language & & 2269 & 97.8 \\
\hline \multicolumn{4}{|l|}{ Education level } \\
\hline Primary/secondary & & 279 & 11.9 \\
\hline$\leq$ Three-years higher education & & 958 & 40.9 \\
\hline > Three-years higher education & & 1105 & 47.2 \\
\hline Deprivation (EPICES Index) & & 409 & 18.1 \\
\hline Cancer history & & 1073 & 45.8 \\
\hline \multicolumn{4}{|l|}{ Difficulties in asking physicians questions } \\
\hline Always (1) & & 37 & 1.6 \\
\hline Often (2) & & 229 & 9.8 \\
\hline Sometimes (3) & & 735 & 31.4 \\
\hline Rarely (4) & & 669 & 28.6 \\
\hline Never (5) & & 632 & 27 \\
\hline Missing values & & 40 & 1.7 \\
\hline Health literacy & Possible range & Mean & SD \\
\hline $\mathrm{FCCHL}$ & $14-70$ & 55.58 & 7.06 \\
\hline Functional dimension & $5-25$ & 18.95 & 4.18 \\
\hline Communicative dimension & $5-25$ & 20.66 & 3.01 \\
\hline Critical dimension & $4-20$ & 15.96 & 3.08 \\
\hline
\end{tabular}

FCCHL Functional, Communicative and Critical Health Literacy

were merged in a single "processing" dimension $(\mathrm{RMSEA}=0.123, \mathrm{CFI}=0.888$ and $\mathrm{TLI}=0.866$ ).

Cronbach's $\alpha$ were $>0.7$ for the overall scale $(\alpha=0.77)$ and subscales $(\alpha=0.79, \alpha=0.74$ and $\alpha=0.77$ for functional, communicative and critical dimensions respectively). Communicative and critical HL were moderately linked together $(r=0.46)$ and weakly correlated with the functional dimension ( $r=0.15$ and $r=0.03$ ).

Scalar invariance was reached for education levels and deprivation, but not for age (Additional file 1: Table S2). However, partial scalar invariance was reached in releasing the fourth threshold of FCCHL2 in the 18-40 yearsold age group. That means that this threshold was significantly lower $(0.049)$ than in the older age groups (0.691), i.e. at the same level of functional HL, older people responded more frequently than younger people that they agreed with the fact that they felt the print was too small for them to read.

Age showed negative associations with FCCHL but not with the communicative dimension (Table 3). When the functional HL or FCCHL score was calculated using the four or thirteen age-invariant items only (without FCCHL2), no association was observed with age ( $p$-value $=0.411$ or $p$-value $=0.053$ ). A higher education level and lack of deprivation were significantly associated with higher levels of HL, except that deprivation was not associated with critical HL. In contrast having French as mother language (97.8\%) was only associated with higher functional HL. No gender differences were observed.

\section{Discussion}

Our results confirmed similar or better psychometric properties of the French version of the FCCHL scale compared with the original version [5].

Consistent with previous studies [5-7], exploratory analysis revealed a 3-factor model confirming the overall structure of the scale, with satisfactory internal consistency of each FCCHL dimension. Similar to results among German citizens showing a 2-factor model combining communicative and critical HL into "processing HL" [8], these two dimensions were the most correlated here. A single item of the critical dimension (FCCHL14) primarily loaded on the communicative dimension. This dual loading might be explained in light of the wording of this item, because 'collect' can reflect accessing information by communication, while 'make' refers more to critical aspects needed for making decisions. As no major differences in fit indices were observed when FCCHL14 was included in the communicative dimension, this item was left in the critical dimension to be more consistent with the original version.

The internal consistency of each FCCHL dimension was satisfactory. Our findings differ slightly from previous findings in Dutch citizens [6] and young Australian cancer patients [9], which found that internal consistency of the communicative dimension was less satisfactory $(\alpha=0.63$ in both studies). These differences may be explained by fewer items $(n=3)$ in the FCCHL-AYAC for the communicative dimension and difficulties answering items reported by Dutch citizens. In the latter study, items seemed to be too abstract and citizens clearly underlined that they did not understand what was meant by 'applying information to their daily life' or by 'considering whether information was applicable'.

As hypothesized and in line with previous studies [4, 21, 22], people with lower education had lower HL compared to people with higher education. Furthermore, socioeconomic deprivation tended to be associated with lower $\mathrm{HL}$, except for the critical dimension as previously reported $[4$, 22]. Only the functional dimension of FCCHL was able to discriminate the few participants who had a mother language other than French highlighting difficulties understanding health information associated with potential difficulties understanding French generally.

The negative association between age and functional HL or FCCHL total score disappeared when those scores 
Table 2 Factor structure of the FCCHL scale. ( $n=781$, Training sample)

\begin{tabular}{|c|c|c|c|c|c|}
\hline & & \multicolumn{3}{|l|}{ Factor } & \multirow[t]{2}{*}{ Communality } \\
\hline & & 1 & 2 & 3 & \\
\hline \multicolumn{6}{|c|}{ Functional health literacy } \\
\hline FCCHL1 & Find characters that I cannot read & 0.75 & -0.01 & 0.08 & 0.66 \\
\hline FCCHL2 & Feel that the print is too small for me to read & 0.59 & -0.09 & -0.08 & 0.43 \\
\hline FCCHL3 & Feel that the content is too difficult for me to understand & 0.93 & 0.03 & 0.02 & 0.88 \\
\hline $\mathrm{FCCHL4}$ & Feel that it takes a long time to read them & 0.70 & 0.02 & -0.01 & 0.50 \\
\hline FCCHL5 & Need someone to help me read them & 0.66 & 0.03 & -0.05 & 0.46 \\
\hline \multicolumn{6}{|c|}{ Communicative health literacy } \\
\hline FCCHL6 & Collect information from various sources & 0.02 & 0.78 & -0.04 & 0.66 \\
\hline FCCHL7 & Extract the information I want & -0.03 & 0.71 & -0.03 & 0.52 \\
\hline FCCHL8 & Understand the obtained information & 0.24 & 0.62 & 0.06 & 0.48 \\
\hline FCCHL9 & Communicate my opinion about my illness & -0.08 & 0.73 & 0.08 & 0.46 \\
\hline FCCHL10 & Apply the obtained information to my daily life & 0.02 & 0.68 & 0.04 & 0.45 \\
\hline \multicolumn{6}{|c|}{ Critical health literacy } \\
\hline FCCHL11 & Consider whether the information is applicable to me & -0.02 & 0.23 & -0.71 & 0.73 \\
\hline FCCHL12 & Consider whether the information is credible & -0.01 & -0.07 & -0.97 & 0.86 \\
\hline FCCHL13 & Check whether the information is valid and reliable & 0.01 & 0.09 & -0.68 & 0.54 \\
\hline FCCHL14 & Collect information to make my healthcare decisions & -0.04 & 0.42 & -0.27 & 0.37 \\
\hline
\end{tabular}

Loading values higher than 0.4 are bolded

were computed without the FCCHL2 item found to be non-invariant across age groups. This is likely due to differing interpretations of this item between younger and older people and highlights that spurious age differences may be observed when FCCHL2 is used. It also questions the association between age and FCCHL that was observed in a previous study [4] and suggests that measurement invariance across age should also be studied for FCCHL versions in other languages, as recommended in the guidelines [23]. In our study, the critical HL score was thus the only one that was significantly negatively associated with age and it might reflect lower empowerment among older people [24]. The lack of impact of age on the communicative dimension was expected [4]. It might also be explained by our recruitment of persons, very involved in research and registered to participate in mainly online surveys, who might not have difficulties communicating no matter their age.

Our study has some limitations. We were unable to compare the translated FCCHL against an objective test of functional HL. Moreover, our sample included mainly women (96\%), who were highly educated and had French as their mother language. However the sample was more heterogeneous regarding age and cancer history and the FCCHL showed variability despite them being negatively skewed (Additional file 1: Figure S1).

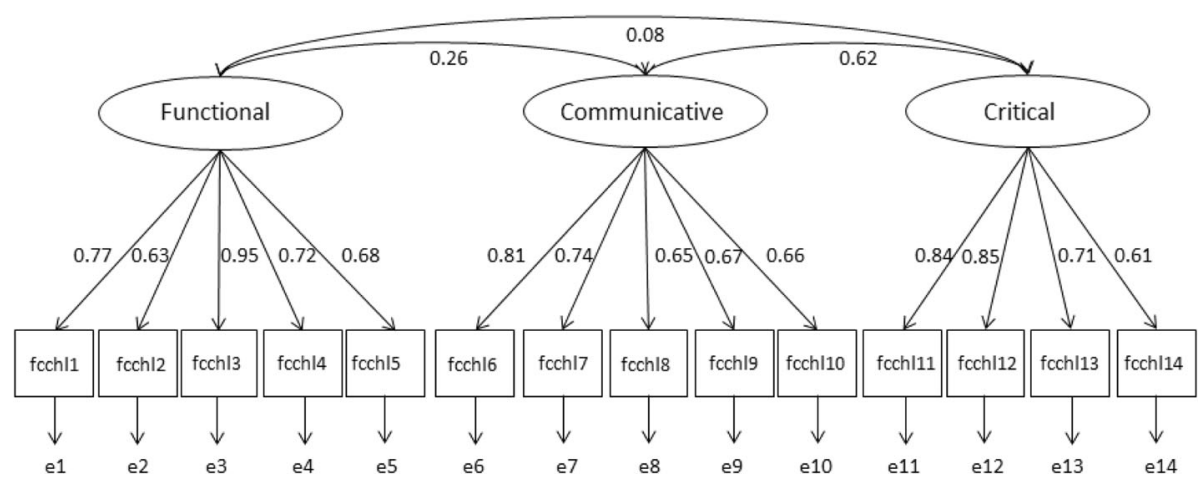

Fig. 1 Standardized parameter estimates for the 3 -factor model of FCCHL ( $n=1561$, validation sample). Rectangles represent the observed variables (items) and ellipses represent the latent constructs (factors). Values on the single-headed arrows leading from the factors to the items are standardized factor loadings. Values on the curved double-headed arrows are correlations between factors terms 
Table 3 Bivariate relationships between FCCHL and sociodemographic characteristics $(n=2342)$

\begin{tabular}{|c|c|c|c|c|c|c|c|c|}
\hline & \multicolumn{2}{|l|}{ Functional $\mathrm{HL}$} & \multicolumn{2}{|c|}{ Communicative $\mathrm{HL}$} & \multicolumn{2}{|l|}{ Critical HL } & \multicolumn{2}{|l|}{ FCCHL } \\
\hline & Mean \pm SD & $\mathrm{P}$ & Mean \pm SD & $P$ & Mean \pm SD & $\mathrm{P}$ & Mean \pm SD & $\mathrm{P}$ \\
\hline \multicolumn{9}{|l|}{ Age } \\
\hline $18-40$ & $19.57 \pm 3.93$ & $<0.001^{\mathrm{a}}$ & $20.63 \pm 2.78$ & 0.617 & $16.32 \pm 2.75$ & $<0.001$ & $56.53 \pm 6.32$ & $<0.001^{\mathrm{b}}$ \\
\hline $41-60$ & $18.70 \pm 4.27$ & & $20.62 \pm 3.12$ & & $15.86 \pm 3.13$ & & $55.20 \pm 7.34$ & \\
\hline $61-83$ & $18.49 \pm 4.27$ & & $20.78 \pm 3.15$ & & $15.60 \pm 3.43$ & & $54.88 \pm 7.38$ & \\
\hline \multicolumn{9}{|l|}{ French maternal Language } \\
\hline No & $17.35 \pm 4.95$ & 0.006 & $20.96 \pm 2.96$ & 0.472 & $16.68 \pm 2.36$ & 0.094 & $55.59 \pm 7.04$ & 0.548 \\
\hline Yes & $18.98 \pm 4.16$ & & $20.65 \pm 3.01$ & & $15.95 \pm 3.09$ & & $55.00 \pm 6.95$ & \\
\hline \multicolumn{9}{|l|}{ Education } \\
\hline Primary/secondary & $17.36 \pm 4.36$ & $<0.001$ & $20.11 \pm 3.30$ & $<0.001$ & $15.57 \pm 3.23$ & $<0.001$ & $53.05 \pm 7.47$ & $<0.001$ \\
\hline$\leq$ Three-years higher education & $18.71 \pm 4.12$ & & $20.48 \pm 2.95$ & & $15.63 \pm 3.15$ & & $54.84 \pm 6.78$ & \\
\hline > Three-years higher education & $19.56 \pm 4.05$ & & $20.94 \pm 2.96$ & & $16.34 \pm 2.94$ & & $56.86 \pm 6.92$ & \\
\hline \multicolumn{9}{|l|}{ Deprivation (EPICES Index) } \\
\hline No & $19.13 \pm 4.08$ & $<0.001$ & $20.73 \pm 3.02$ & 0.003 & $15.97 \pm 3.06$ & 0.721 & $55.84 \pm 7.00$ & $<0.001$ \\
\hline Yes & $18.29 \pm 4.42$ & & $20.25 \pm 2.98$ & & $15.91 \pm 3.17$ & & $54.46 \pm 7.02$ & \\
\hline
\end{tabular}

HL Health Literacy, FCCHL Functional, Communicative and Critical Health Literacy

${ }^{a} p$-value $=0.411$ if functional $\mathrm{HL}$ score computed without $\mathrm{FCCHL} 2$, item found to be non-invariant across age groups

${ }^{\mathrm{b}} p$-value $=0.053$ if FCCHL score computed without FCCHL2, item found to be non-invariant across age groups

FCCHL also presented good acceptability, as indicated by low levels of missing data, which might be attributable to respondents' high HL.

In this first validation study of a self-report multidimensional health literacy scale in France, the French version of the FCCHL demonstrated adequate reliability and validity among cancer patients and general population. It highlighted that measurement invariance across age should be studied more systematically when validating HL measures. Further studies are needed to examine the French FCCHL stability among less educated, less literate samples including more men and those without French as their mother language. This relatively brief measure could be used among both patients and general population to allow identification of people with low $\mathrm{HL}$ in order to develop this skill and to tailor health communication accordingly.

\section{Additional file}

Additional file 1: Table S1. Distribution of the responses to the different items on the questionnaire and polychoric correlations between the various FCCHL items $(n=2342)$. Table S2. Measurement invariance across age groups, education levels, and deprivation. Figure S1. Distribution of FCCHL score and subscores. (DOCX $77 \mathrm{~kb})$

\section{Acknowledgements}

We particularly thank all members of Seintinelles association. We are also grateful to Cyril Berenger for his help to implement the e-survey.

\section{Funding}

The project leading to this publication has received funding from Excellence Initiative of Aix-Marseille University - A*MIDEX, a French "Investissements
d'Avenir" program. This work was supported by the French National Cancer Institute (grant number INCA_8102); IRESP (grant number RINGA-AAP16-HSR-13) for French teams collaboration; the funding partners of the IRESP in the framework of the "2016 call for general project Health services".

\section{Authors' contributions}

All authors of this research paper have made substantial contributions to all of the following: (1) the conception and design of the study, or acquisition of data, or analysis and interpretation of data, (2) drafting the article or revising it critically for important intellectual content. All authors have read and approved the final version to be submitted.

\section{Ethics approval and consent to participate}

This study has been approved by the Inserm Ethics Committee (IRB00003888, N $\left.{ }^{\circ} 15-266\right)$.

\section{Competing interests}

The authors declare that they have no competing interests.

\section{Publisher's Note}

Springer Nature remains neutral with regard to jurisdictional claims in published maps and institutional affiliations.

\section{Author details}

1Aix-Marseille Univ, INSERM, IRD, UMR912, SESSTIM, Institut Paoli-Calmettes, "Cancers, Biomedicine \& Society" group, 232, Bd Ste Marguerite, BP 156, 13273 Marseille Cedex 9, France. ${ }^{2}$ Public Health and Epidemiology Department, APHP, Bicêtre Hospital, Le Kremlin-Bicêtre, France. ${ }^{3}$ Université Paris-Saclay, Univ. Paris-Sud, UVSQ, CESP, INSERM, Le Kremlin Bicêtre, France. ${ }^{4}$ Centre for Oncology Education and Research Translation (CONCERT), Ingham Institute for Applied Medical Research \& South Western Sydney Clinical School, University of New South Wales, Liverpool, NSW, Australia. ${ }^{5}$ Psycho-Oncology Co-operative Research Group (PoCoG), School of Psychology, University of Sydney, Sydney, Australia. ${ }^{6}$ APHM, Timone Hospital, Public Health Department (BIOSTIC), Marseille, France. 
Received: 9 August 2017 Accepted: 4 January 2018

Published online: 07 February 2018

\section{References}

1. Sørensen, K., Van den Broucke, S., Fullam, J., Doyle, G., Pelikan, J., Slonska, Z., et al. (2012). Health literacy and public health: A systematic review and integration of definitions and models. BMC Public Health, 12(1), 80.

2. Nielsen-Bohlman, L., \& Institute of Medicine (Eds.). (2004). Health literacy: A prescription to end confusion. Washington, DC: National Acad. Press.

3. Nutbeam, D. (2000). Health literacy as a public health goal: A challenge for contemporary health education and communication strategies into the $21 \mathrm{st}$ century. Health Promot Int, 15(3), 259-267.

4. Ishikawa, H., Takeuchi, T., \& Yano, E. (2008). Measuring functional, communicative, and critical health literacy among diabetic patients. Diabetes Care, 31(5), 874-879.

5. Suka, M., Odajima, T., Kasai, M., Igarashi, A., Ishikawa, H., Kusama, M., et al. (2013). The 14-item health literacy scale for Japanese adults (HLS-14). Environ Health Prev Med, 18(5), 407-415.

6. van der Vaart, R., Drossaert, C. H. C., Taal, E., ten Klooster, P. M., HilderinkKoertshuis, R. T. E., Klaase, J. M., \& van de Laar, M. A. F. J. (2012). Validation of the Dutch functional, communicative and critical health literacy scales. Patient Educ Couns, 89(1), 82-88.

7. Fransen, M. P., Van Schaik, T. M., Twickler, T. B., \& Essink-Bot, M. L. (2011). Applicability of internationally available health literacy measures in the Netherlands. J Health Commun, 16(Suppl 3), 134-149.

8. Dwinger, S., Kriston, L., Härter, M., \& Dirmaier, J. (2015). Translation and validation of a multidimensional instrument to assess health literacy. Health Expect, 18(6), 2776-2786.

9. McDonald, F. E. J., Patterson, P., Costa, D. S. J., \& Shepherd, H. L. (2016). Validation of a health literacy measure for adolescents and young adults diagnosed with cancer. J Adolesc Young Adult Oncol, 5(1), 69-75.

10. Husson, O., Mols, F., Fransen, M. P., van de Poll-Franse, L. V., \& Ezendam, N. P. M. (2015). Low subjective health literacy is associated with adverse health behaviors and worse health-related quality of life among colorectal cancer survivors: Results from the profiles registry. Psycho-Oncology, 24(4), 478-486.

11. Fasse, L., Flahault, C., Vioulac, C., Lamore, K., Van Wersch, A., Quintard, B., \& Untas, A. (2017). The decision-making process for breast reconstruction after cancer surgery: Representations of heterosexual couples in long-standing relationships. Br J Health Psychol, 22(2), 254-269.

12. Rouquette, A., \& Falissard, B. (2011). Sample size requirements for the internal validation of psychiatric scales. Int J Methods Psychiatr Res, 20(4), 235-249.

13. Terwee, C. B., Bot, S. D. M., de Boer, M. R., van der Windt, D. A. W. M., Knol, D. L., Dekker, J., et al. (2007). Quality criteria were proposed for measurement properties of health status questionnaires. J Clin Epidemiol, 60(1), 34-42.

14. de Vet, H. C. W., Terwee, C. B., Mokkink, L. B., \& Knol, D. L. (2011) Measurement in medicine: A practical guide. Cambridge: University Press.

15. Bland, J. M., \& Altman, D. G. (1997). Statistics notes: Cronbach's alpha. BMJ, 314(7080), 572.

16. Brown, T. A. (2006). Confirmatory factor analysis for applied research (methodology in the social sciences).

17. Muthén, B., \& Muthén, B. O. (2009). Statistical analysis with latent variables. New York: Wiley.

18. Hu, L., \& Bentler, P. M. (1999). Cutoff criteria for fit indexes in covariance structure analysis: Conventional criteria versus new alternatives. Struct Equ Model Multidiscip J, 6(1), 1-55.

19. Chen, F. F. (2007). Sensitivity of goodness of fit indexes to lack of measurement invariance. Struct Equ Model Multidiscip J, 14(3), 464-504.

20. Cheung, G. W., \& Rensvold, R. B. (2002). Evaluating goodness-of-fit indexes for testing measurement invariance. Struct Equ Model Multidiscip J, 9(2), 233-255.

21. Nutbeam, D. (2008). The evolving concept of health literacy. Soc Sci Med, 67(12), 2072-2078.

22. Paasche-Orlow, M. K., Parker, R. M., Gazmararian, J. A., Nielsen-Bohlman, L. T., \& Rudd, R. R. (2005). The prevalence of limited health literacy. J Gen Intern Med, 20(2), 175-184.

23. Mokkink, L. B., Terwee, C. B., Knol, D. L., Stratford, P. W., Alonso, J., Patrick, D. L., et al. (2010). The COSMIN checklist for evaluating the methodological quality of studies on measurement properties: A clarification of its content. BMC Med Res Methodol, 10, 22.

24. Bynum, J. P. W., Barre, L., Reed, C., \& Passow, H. (2014). Participation of very old adults in health care decisions. Med Decis Mak, 34(2), 216-230.

\section{Submit your manuscript to a SpringerOpen ${ }^{\circ}$ journal and benefit from:}

- Convenient online submission

- Rigorous peer review

- Open access: articles freely available online

- High visibility within the field

- Retaining the copyright to your article

Submit your next manuscript at springeropen.com 